\title{
Wilhelm Freiherr v. Stauffenberg $\dagger$.
}

Wilhelm Schenk, Freiherr zu Stauffenberg, ist geboren 1879 als Sohn des Majors Freiherrn Carl zu Stauffenberg in Augsburg. In seinem 8. Lebensjahre erkrankte er an einer schweren eitrigen Rippenfellentzündung, die nach Monaten durch eine Rippenresektion geheilt wurde, aber eine dauernde Schrumpfung der einen Lunge und eine Verkrümmung des Brustkorbes und der Wirbelsäule hinterließ. Dieses körperliche Leiden, welches ihm unter seinen Altersgenossen eine Ausnahmestellung auferlegte, begründete bei dem sensitiv veranlagten Knaben eine Verinnerlichung und Vergeistigung. Früh lernte er mehrere fremde Sprachen beherrschen und seine künstlerischen Neigungen zu betätigen. Von außerordentlichem Einfluß war auf ihn sein Onkel, der bekannte, hochgebildete Politiker und Reichstagsabgeordnete Franz Freiherr v. Stauffenberg, auf dessen Gut er seine Ferien verbrachte. Dieser unterstützte sein Interesse an Büchern, indem er ihm seine reichhaltige Schloßbibliothek zur Verfügung stellte, erweckte und schärfte seine kritische Begabung. - Nach Vollendung der Gymnasialstudien in Augsburg und München, wandte sich Wilhelm v. Stauffenberg, der sich zum Gutsbesitzer bestimmt glaubte, dem Studium der Rechte zu, da er aber zu diesem keine innerliche Verwandtschaft fühlte, ging er bauptsächlich seinen künstlerischen Neigungen nach und vertiefte sich in das Studium der Philosophie. Immerhin wurde eine als Doktordissertation projektierte Untersuchung über die Todesstrafe so gut wie vollendet. Während der letzten Semester seines juristischen Studiums wurde es ihm klar, daß er eines Berufes bedürfe, der ihm den Einsatz und die Entfaltung seiner ganzen Persönlichkeit ermögliche, und sein brennendes Interesse für den Menschen, seine Eigenarten und Gemütszustände führten ihn zu der Medizin. Hier auf dem Gebiet der Naturwissenschaften fühlte er sich sofort zu Hause. In Bonn machte Pflüge rs gewaltige Persönlichkeit großen Eindruck auf ihn. In München und Tübingen wurden die klinischen Semester vollendet. So sehr ihn dabei auch die rein wissenschaftliche Seite des Faches und besonders der Neurologie fesselte, so wandte sich doch seine innerliche herzens- 
warme Teilnahme von Anfang an hauptsächlich dem leidenden Menschen zu. Nach Absolvierung des Staatsexamens 1907 promovierte er mit einer Schrift über „Zwei Fälle von Hemianästhesie ohne Motilitätsstörung“. Im Jahre 1909 trat er als Assistenzarzt an der 2. Med. Klinik ein, der er bis zu seinem Lebensende angehörte. Wenn er sich in seiner Assistententätigkeit auch mit dem ganzen weitem Gebiet der inneren Medizin zu beschäftigen hatte, so galten doch seine Neigungen und seine Forscherarbeit hauptsächlich den Erkrankungen des Gehirns und der Nervenkrankheiten überhaupt. Zur weiteren Vertiefung in dieses Spezialstudium wandte er sich zuerst nach Berlin und da ihm O p pe nhei m s sorgfältige Art der einfachen Registrierung der Beobachtungen kein völliges Genüge bot, so ging er 1909 nach Paris. Dort hörte er vor allem Dé je ri ne, Pierre Marie und die anderen Kliniken der Salpêtrière. Die französische Art, die Probleme aufzufassen, war ihm sehr anregend, der Kampf der alten Neurologie, Charcotschen Stils mit der neueren Lokalsationslehre war entbrannt und wurde mit großer Heftigkeit geführt. Neben dem medizinischen Studium wurde auch Philosophie getrieben und u. a. Berg son gehört, mit dessen „Matière et mémoire“ sich v. Sta uffen berg in seinen späteren Arbeiten immer wieder auseinandersetzte. Reich an neuen Eindrücken kehrte er an die Münchner Klinik zur ück, und nun galt es, sich der selbständigen Forscherarbeit zu widmen. v. Sta uffe nberg begann damit, die organischen Gehirnkrankheiten, u. a. einige Fälle von Seelenblindheit einem eingehenden analytischen Studium zu unterwerfen; die Verarbeitung des pathologisch-anatomischen Befundes setzt eine gründliche Beherrschung der Technik und der feineren Gehirnanatomie voraus, und da an der Münchner Klinik nicht die ger ügende Anleitung für gehirnanatomische Studien gegeben war, ging v. S ta uffe nberg auf den Rat seines Klinikchefs 1912 nach Zürich zu v. Monakow. Dort fand er, was er suchte: nicht bloß den Meister der hirranatomischen Forschurg, sondern auch neue umwälzende Ideen, welche ihm deshalb so sympathisch waren, weil v. Mo nakow den engen Standpunkt der alten Lokalisationslehre verlassen und auf dem Boden der Biologie und Psychologie eine neue Auffassung der Gehirnfunktionen eir geführt hatte. In Zürich wurde der anatomische Teil von v. S ta uffe n be rg s Arbeit über die Seeler blirdheit vollendet, die er rach seiner Rückkehr als Habilitatior sschrift 1913 der Münchner Fakultät einreichte. Diese umfangreiche Arbeit hat die älteren einfachen Arschaurgen über das Wesen dieser eigerartigen Erkrankurg nech allen Richtur gen er weitert und vertieft und wird grundlegend bleiben, nicht nur in anatomischer Beziehurg; sondern auch durch die Einführurg der psychologischen Betrachturgsweise in die Untersuchung der Kranken. Auf demselben Boden bewegten sich auch v. Sta uffenbergs weitere Forschungen über die aphasischen, agnosti- 
schen und apraktischen Symptome, welche kurz vor seinem Tode in dieser Zeitschrift ersehienen sind und jahrelange Studien, sowie viele Serienreihen von großen Gehirnschnitten zur Grundlage hatten. In allen diesen Schriften kommt die vorurteilsfreie, kritische und dabei doch künstlerische, philosophisch durchgebildete Denkungsart $v$. Sta uffe nbergs zur vollen Geltung. Den Schluß von v. Stauffenbergs hirnanatomischen Arbeiten bilden zwei Fälle von Erkrankungen des extrapyramydalen motorischen Systems und von diffuser periaxialer Encephalitis. Die prächtigen Schnittserien bilden einen besonderen Schatz der reichen Sammlung, welche v. S ta uffe nberg seiner Klinik hinterlassen hat.

In Zürich hat $v$. Stauffenberg aber noch andere Anregungen gefunden, und zwar solche, welche in seinem Wesen besonders anklargen und die für ihn in der Folgezeit mehr und mehr bestimmend werden sollten: Thn, den scharfen Beobachter und ausgezeichneten Menschenkenner interessierte nicht so sehr „,der Fall“" mit seinen grob nachweisbaren Defekten, ihn interessierte leidenschaftlich der $\mathrm{kranke}$ Mensch mit seinen Charakterveränderungen und der Wandlung seiner emotionellen Reaktionsweise. In Zürich schloß er sich an Ble ule $\mathbf{r}$ an, um näheren Einblick in die Psychiatrie und namentlich das Wesen der Schizophrenie zu gewinnen, und dann führte ihn sein Wunsch, dem seelisch-kranken Menschen zu helfen, auf das Gebiet der Psychotherapie. Obwohl er den Lehren Freuds ursprünglich skeptisch, ja ablehnend geger über stand, gewann er doch im näheren Verkehr mit Verag u th, Frank und Jung ein steigendes Interesse an der neueren psychotherapeutischen Richtung. Ihr galten nach seiner Rückkehr an die Münchner Klinik seine hauptsächlichen Bestrebungen und er vertrat sie auch in seinen Vorlesur gen. Er erkannte bald, daß die Methode des Abreagierenlassens von Affekten meist nicht gen ügt, um der krankhaften Erscheinungen Herr zu werden und die psychoanaly tische Behandlungsmethode der zugrunde liegenden Konflikte schien ihm mehr Aussicht auf Erfolg zu versprechen. Mit welch unermüdlicher Liebe zum kranken Menschen hat er sich seit jenen eindrucksvollen Züricher Jahren der psychotherapeutischen Kleinarbeit hingegeben und schließlich doch mit wie geringem Erfolg! Das dankbare Vertrauen, das ihm seine Patienten und auch seine Schüler entgegenbrachten, waren der Lohn für seine aufopfernde Tätigkeit, welche seine Kräfte mehr und mehr verzehrten. In seinen Vorlesungen hat er sich auf das Gebiet der Neurologie und speziell der Gehirnkrankheiten beschränkt und stets einen ausgewählten Kreis von Zuhörern um sich versammelt. G ößer war noch sein persönlicher Einfluß auf die einzelnen, ihm näher stehenden Schüler auf der Krankenabteilung. 
Welcher Verlust für die Medizin, daß ein so hochbegabter, tief gebildeter Geist mitten aus seiner reifsten Entwicklung dahingerafft wurde! Für die Münchner 2. medizinische Klinik bedeutete v. S ta uf fe nbergs Tod, der am 13. II. 1918 an einer bösartigen Lungenentzündung erfolgte, eine unersetzliche Lücke. Wilhelm v. Sta uffe nberg war ein Edelmann im vollen Sinn des Wortes, er war trotz aller Entsagung, die ihm sein körperliches Leiden auferlegte, ein glücklicher Mensch, denn es fehlte ihm ganz die Fähigkeit, an sich selbst zu denken.

F. Müller (München). 